\title{
Method to Separate In-Plane and Out-of-Plane Misorientation From the Total Misorientation at Grain Boundaries in Polycrystalline YBCO Films
}

\author{
J. Li, ${ }^{*}$ and A. Goyal*
}

* Metal and Ceramics Division, Oak Ridge National Laboratory, P.O. Box 2008, MS 6116, Oak Ridge, TN 37831

It is well established that grain boundary $(\mathrm{GB})$ misorientation determines the transport properties of $\mathrm{YBa}_{2} \mathrm{Cu}_{3} \mathrm{O}_{7}(\mathrm{YBCO})$ superconducting films. Hence it is of interest to accurately determine GB misorientation in thick YBCO films in coated conductors which are of interest for many electric power applications. Furthermore, it is of interest to determine different components of the total misorientation at a GB. In this paper we report on a methodology to separate the in-plane and outof-plane misorientation components from the total misorientation at a GB in YBCO films.

Fig. 1a shows the transmission electron microscopy (TEM) image for YBCO cross-section sample with [100] direction and (001) film plane parallel to electron beam. All of the grains A, B, C, D, E, $\mathrm{F}, \mathrm{G}, \mathrm{H}$. I with $200 \mathrm{~nm}$ sizes are oriented at [100] but have small angle misorientation and show different contrast. Fig. $1 \mathrm{~b}$ shows the global coordinate system $(\mathrm{X}, \mathrm{Y}, \mathrm{Z})$. The $\alpha, \beta$ and $\gamma$ angles represent the misorientation of each YBCO grain (with $\boldsymbol{a}, \boldsymbol{b}, \boldsymbol{c}$ axes system) in respect to the global coordinate system. The $\alpha, \beta$ angles are refereed to as "out-of-plane" misorientation, and the $\gamma$ angle is refereed to as "in-plane" misorientation, respectively. For two adjacent YBCO grains, we only need to determine the relative misorientation angle $\theta$ between two grains, then get $\alpha$-, $\beta$ - (out-ofplane), and $\gamma$ - (in-plane) misorientation components. Where the $\alpha$-component is easy to be determined directly from the [100] diffraction patterns of two adjacent YBCO grains as the angle between the $(00 l)$ reflection directions of two grains.

To determine the $\beta$ - and $\gamma$ - components, convergent beam electron diffraction (CBED) patterns were recorded for each grain. Due to the misorientation, the Ewald reflecting sphere has different intersection with the (100)* reciprocal plane for different grains; this will be shown as a bright "arc" with different feature on [100] CBED patterns. Fig. 2a, Fig. 2b show two examples. Upon the reciprocal space geometry, by matching the arc in each CBED pattern with a circle and finding the circle center position $\mathrm{O}\left(\mathrm{O}_{1}\right.$ and $\mathrm{O}_{2}$ in Fig. 2a, Fig. 2b), we can get the total misorientation angle $\theta$ between two YBCO grains with the circle centers $\mathrm{O}_{1}$ and $\mathrm{O}_{2}$ by: $\theta=O_{1} O_{2} / L$, and get the $\beta$ - and $\gamma$ components by: $\beta=O_{1} O_{2} \cos \varphi / L, \gamma=O_{1} O_{2} \sin \varphi / L$. Where $\mathrm{L}$ is the TEM camera length and $\varphi$ is the angle between the line $\mathrm{O}_{1} \mathrm{O}_{2}$ and $(00 l)$ reflection direction of YBCO (See Fig. 3). The misorientation angles and in-plane and out-of-plane components between each adjacent YBCO grain pair have been determined for the grains A-I in Fig. 1. The result indicated that most of the grain boundaries have misorientation angles $\theta<5^{\circ}$ with the in-plane component $\gamma<3^{\circ}$ and out-of-plane components $\alpha<1^{\circ}$ and $\beta<2^{\circ}$. This will provide an insight into explanation of the properties of this material.

Reference

[1] Research sponsored by the USDOE, under contract DE-AC05-00OR22725. 


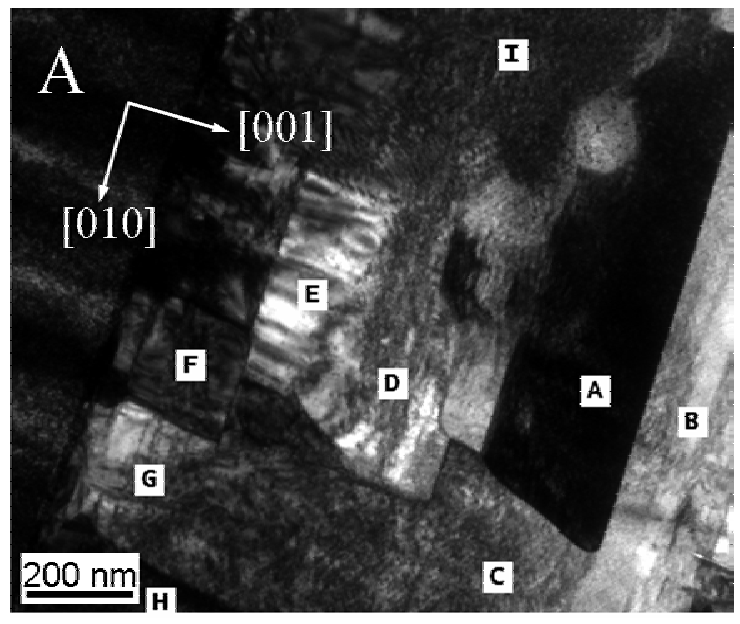

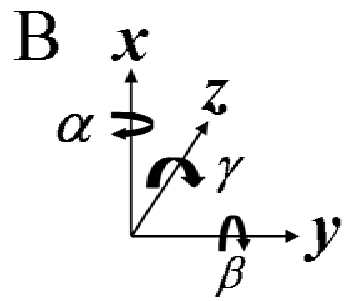

Global coordinate system

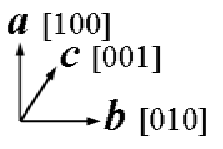

YBCO axes

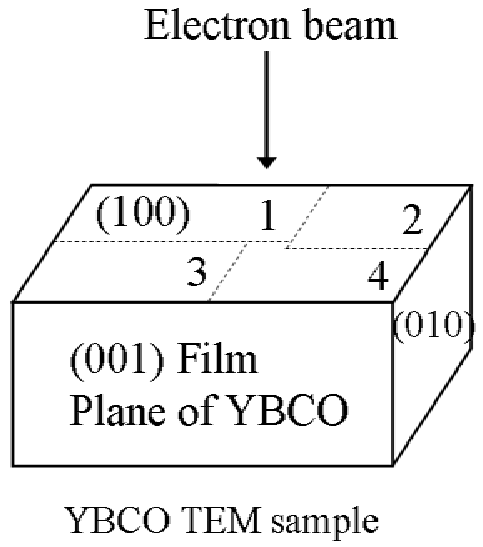

Fig. 1. A. TEM image for YBCO cross-section sample oriented in [100]. B. $\alpha, \beta$ and $\gamma$ angles represent misorientation angles of YBCO grains (with $\boldsymbol{a}, \boldsymbol{b}, \boldsymbol{c}$ axes system) in respect to the global coordinate system $(\mathrm{X}, \mathrm{Y}, \mathrm{Z})$.
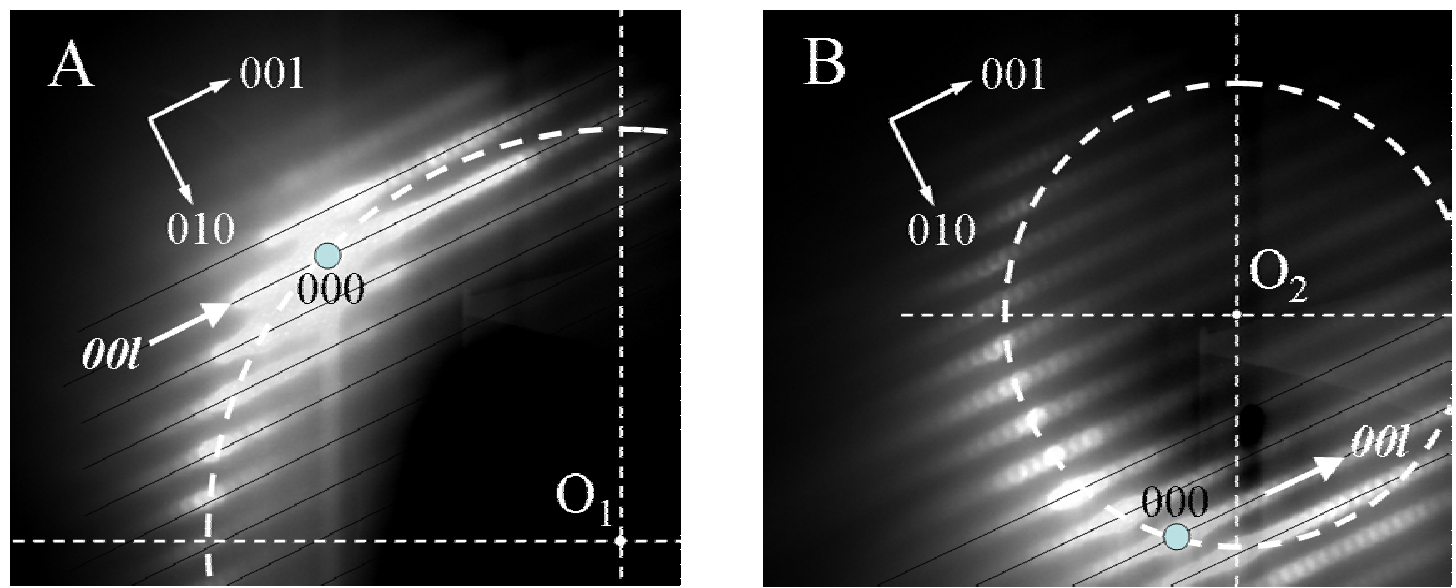

Fig.2. Bright "arc" with different feature on the [100] CBED patterns (A, B) of two adjacent YBCO grains.

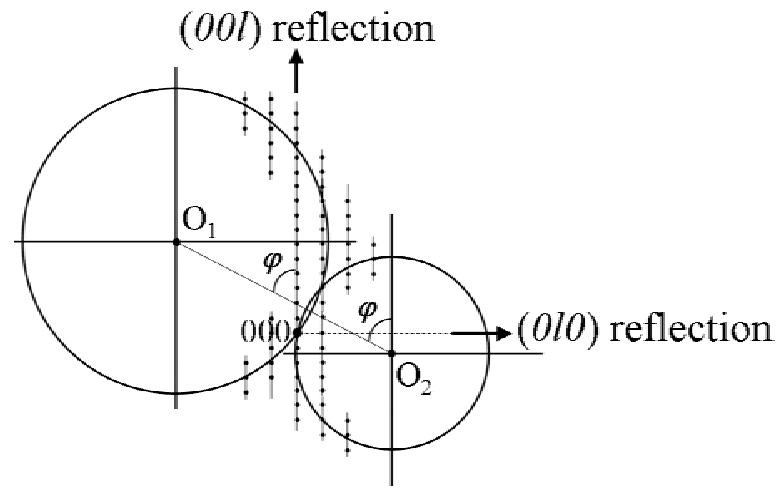

Fig. 3. Measurement of misorientation angle $\theta$ and $\beta$ - and $\gamma$-components between two adjacent YBCO grains by using reciprocal space geometry. 